\title{
Sensing Opportunities in UMTS spectrum
}

\author{
Work in progress \\ Paulo Marques ${ }^{1,2}$ and Atílio Gameiro ${ }^{1}$ \\ ${ }^{1}$ Universidade de Aveiro, Instituto de Telecomunicações, DETUA, 3810 Aveiro, Portugal. \\ ${ }^{2}$ ESTCB, Instituto Politécnico de Castelo Branco, 6000 Castelo Branco, Portugal \\ E-mails: pmarques@av.it.pt and atilio@av.it.pt
}

\begin{abstract}
The UMTS radio frequency spectrum is a highly expensive commodity. While the UMTS standards make very efficient use of the allocated bands there is however opportunity for further advancements. This paper focuses on opportunistic use of the UMTS spectrum as a means of ensuring that the maximum possible use of this valuable resource is made. In particular we focus on the local detection of UMTS TDD signals through the use of a cyclostationary feature detector. Simulation results for the use of this detector in the presence of multipath propagation and shadowing effects are presented.
\end{abstract}

Keywords-sensing, UMTS, cyclostationarity

\section{Introduction}

It is expected that the demand for wireless services will continue to increase in the near and medium term, calling for more capacity and putting more and more pressure on the spectrum availability. While the use of advanced signal processing techniques may enable a very efficient usage of the spectrum even in the traditional framework of command and control spectrum policy, there is a worldwide recognition that these methods of spectrum management have reached their limit and are no longer optimal and new paradigms must be sought [1]. In fact independent studies carried out in different places (e.g. [2]) have shown that most of the assigned spectrum is under-utilized. Thus the problem is in most cases a problem of inefficient spectrum management rather than spectrum shortage. The development of frequency agile terminals that can sense "holes" in the spectrum and adapt their transmission characteristics to use these "holes" may provide one tool to address and take advantage of this spectrum underutilization. The evidence of the change and evolution in the approaches of spectrum management can already be seen in the development of the IEEE 802.22 cognitive radio based standard for fixed, point-to-multipoint, wireless regional area networks that operates on unused channels in the TV VHF/UHF bands between 54 and $862 \mathrm{MHz}$ on a noninterfering basis [3]. The detecting of "holes" and the subsequent use of the unoccupied spectrum is referred to as opportunistic use of the spectrum. An Opportunistic Radio (OR) is the term used to describe a radio that is capable of such operation [4].
In this paper we consider the sensing component for an opportunistic radio that shares the band with an UMTS network. This is motivated by the fact that UMTS radio frequency spectrum has become, in a significant number of countries, a very expensive commodity, and therefore the opportunistic use of these bands could be one way for the owners of the licenses to make extra revenue. In the UK, for example, a sum equivalent to US\$ 154 per citizen has been paid for 2x15 MHz radio frequency license for UMTS [5].

The UMTS Terrestrial Radio Access (UTRA) consists of two modes, a frequency division duplex (FDD) mode and a time division duplex (TDD). The basic radio interface parameters are harmonized between the FDD and TDD modes. It is used a direct sequence spread spectrum modulation with $5 \mathrm{MHz}$ bandwidth and the frame duration is $10 \mathrm{~ms}$ [6].

While the main investments in UMTS networks are currently for FDD mode operation, over 120 of the Europe's and Asia's largest mobile operators have spectrum that is specifically allocated to 3G TDD technologies. In this paper we focus on the opportunistic use of UMTS TDD licensed spectrum, which includes both the $1900-1920 \mathrm{MHz}$ and the $2010-2025 \mathrm{MHz}$ bands [7]. The TDD mode supports duplexing by allocating time slots in a common frequency band. The TDD mode is suited to micro and picocell environments and for wireless local loop applications. TDD facilitates an efficient use of unpaired spectrum and supports data rates up to $2 \mathrm{Mbit} / \mathrm{s}$ using QPSK modulation. Despite this efficient usage there is however opportunity for further advancements. Picocells are used mainly within buildings and are therefore often shielded by walls. This results in a natural limitation of the coverage area and consequently, it is possible to envisage some sets of 5 $\mathrm{MHz}$ spectrum opportunities in the spatial dimension for opportunistic use of the spectrum.

In either mode, the signal transmission in UMTS is based on direct sequence spread spectrum techniques, which poses additional challenges when detecting the spectral opportunities. As the power is spread over a bandwidth significantly higher than the one needed with a non spread signalling scheme, efficient detection techniques ensuring a very small probability of miss detection and, in this way, to avoid the "hidden terminal" problem [3], will have to explore the signal features 
of spread signals. Some work on the reliable detection of spread spectrum signals has been carried out. In [8] the detection of spread spectrum signals using the classical radiometric approach is analysed and in [9] the theoretical limit for the lowest signal-to-noise ratio (SNR) that the radiometer can detect is formulated. In order to overcome radiometer limitations Gardner in [10] exploits cyclostationary features for PSK spread spectrum signal detection for interception applications. More recently, to increase the probability of licensed user detection, some authors [11] [12] have suggested cooperative spectrum sensing techniques where different ORs collaborate by sharing their local sensing information.

In this paper we focus on the local sensing of UMTS TDD signals. As a basic assumption we do not suppose any cooperation between the primary users (UMTS TDD) and the OR network. The paper is organised as follows: In Section 2 the sensing requirements and the metrics associated with the sensing process are discussed. In this section the cyclostationary detector is also introduced. In Section 3 the performance of the cyclostationary detector and its ability to detect UMTS TDD signals is evaluated through a range of simulations. Finally, in Section 4 conclusions are made and future challenges highlighted.

\section{Sensing}

Because of the varying conditions of the channel due to multipath propagation and shadowing, spectrum sensing can become extremely difficult. In this section the metrics and requirements for sensing are defined.

\section{A. Metrics and Requirements}

The key issues in the opportunistic use of spectrum are the ability to accurately detect when spectrum is available and quickly release spectrum on the return of the primary users. The detection of whether the spectrum is currently occupied by a primary user can be stated in terms of a binary hypothesis test, in which the null hypothesis $H_{0}$ corresponds to the absence of a signal (of the primary user) and the alternative hypothesis $H_{1}$ corresponds to the signal presence. If $x(t)$ is the signal received by the OR and $y(t)$ is the primary user's signal then the following equation shows the two hypotheses,

$$
x(t)= \begin{cases}w(t), & H_{0} \\ y(t)+w(t), & H_{1}\end{cases}
$$

In the above equations $w(t)$ is the additive white Gaussian noise (AWGN) with zero mean and variance $\sigma_{n}^{2}$. Considering $d$ as a decision statistic, by definition, the probability of detection $(P d)$ and false alarm $(P f a)$ are given by,

$$
P d=P\left\{d>T H \mid H_{1}\right\} \quad, \quad P f a=P\left\{d>T H \mid H_{0}\right\}
$$

where $T H$ is a suitably chosen threshold level. There is a fundamental tradeoff between the probability of a missed detection, given by $P m=1-P d$, and the probability of a false alarm, $P f a$. This tradeoff has different implications for the OR operation. A high Pm would result in missing the presence of the primary user with a high probability which in turn would increase interference to other primary users. On the other hand, a high Pfa results in low spectrum utilization since false alarms increase the number of wasted opportunities. In the case where the $\mathrm{OR}$ is already in communication, a false alarm decision initiates an unnecessary hop to another frequency. The appropriate value of these metrics are not still clear defined, but in [13] it is suggested that a $P d=99.9 \%$ need to be assured to license owners, otherwise, licensed users are not likely to be willing to share their spectrum with others. Intuitively $P f a$ is independent of the SNR, since under the $H_{0}$ hypothesis there is no primary signal present. On the other hand, $P d$ is dependent on the SNR of the received signal, which also depends on the radio channel conditions. The performance of a specific detection technique may be characterized through Receiver Operation Characteristics (ROC) curves, namely a plot of $P d \mathrm{vs}$. $P f a$.

The observation time, Tobs, required to guarantee a sufficiently low $P m$ is a crucial metric to evaluate the ability of OR's ability not to disturb the primary user operation. The last requirement of note is the computational complexity of the detection algorithm.

\section{B. The Energy Detector}

The energy detector is a well-known technique to identify signal levels. The energy detector measures the received energy for selected time and frequency intervals and assumes that the signal plus noise $\left(H_{1}\right)$ has more energy than the noise only $\left(H_{0}\right)$. The greatest advantage of the energy detector relies on its low complexity. However there are several drawbacks [9]. The most important one is the fact that the energy detector needs to estimate the noise power level before making decisions as to the absence or presence of signals. Realistic limitations of the detector's knowledge of the noise level power produce serious degradation in the energy detector performance. In the UMTS case, due the low Power Spectrum Density (PSD) of spread spectrum signal, the signal presence causes a very small fractional increase in the total energy, thus, uncertainly in the measurement of the noise seriously degrades the radiometer performance, even those as small as a fraction of a $\mathrm{dB}[8]$.

\section{The Cyclostationary Detector}

The detection can obviously be improved if instead of a single parameter one exploits other features and characteristics of communication signals. Man made modulated signals are in general coupled with sine wave carriers, pulse trains, spreading codes, cyclic prefixes, etc., which result in built-in periodicity. Even though in digital communications the data symbol sequences may be in a large number of cases characterized as a discrete stationary random process, the continuous modulated signals are characterized as cyclostationary, since their statistics, mean and autocorrelation, 
exhibit periodicity. This periodicity is typically a feature of man made signals so that a receiver or sensor can exploit it for detection of a random signal with a particular modulation type in a background of noise and other modulated signals. The most common analysis tools for stationary random signals rely on the second order statistics, i.e. are based on the autocorrelation function and the PSD, and it is well known that for such signals non-overlapping frequency bands are uncorrelated. On the other hand, the periodicity inherent in cyclostationary signals implies some spectral redundancy which results in correlation between non-overlapping spectral components separated by some multiple of the cycles [14]. By analogy with the definition of conventional autocorrelation, one can define a spectral correlation function,

$R_{x}(\tau, \alpha)=\lim _{\text {Tobs } \rightarrow \infty} \frac{1}{\text { Tobs }} \int_{-T o b s / 2}^{\text {Tobs } 2} x\left(t+\frac{\tau}{2}\right) x *\left(t-\frac{\tau}{2}\right) e^{-j 2 \pi \alpha t} d t$

where the new parameter $\alpha$ is known as the cycle frequency. When $\alpha$ is set to zero, we get the particular case of the energy detector, and for stationary signals $R_{x}(\tau, \alpha)=0 \forall \alpha \neq 0$. The Spectrum Cyclic Density (SCD) is a Fourier transform of the cyclic autocorrelation, given by,

$S_{x}(f, \alpha)=\int_{-\infty}^{\infty} R_{x}(\tau, \alpha) e^{-j 2 \pi f \tau} d \tau$

If the received signal $y$ and the noise $w$ are uncorrelated

$S_{x}(f, \alpha)=S_{y}(f, \alpha)+S_{w}(f, \alpha)$

Assuming that the additive noise is as a stationary process, then its SCD tends to zero for $\alpha \neq 0$ as Tobs is made infinitely large,

$$
\lim _{T o b s \rightarrow \infty} S_{w}(f, \alpha \neq 0)=0
$$

Unlike the PSD which is a real-valued one dimensional transform, the $\mathrm{SCD}$ is a two dimensional transform. The distinctive characteristic of spectral redundancy makes signal selectivity possible, thus, overlapping features in the power spectrum density are not overlapping feature in the cyclic spectrum, i.e. $\alpha$ domain.

The performance of the cyclic detector is proportional to the strength of the cyclic feature associated with the particular value of $\alpha$. For instance, with PSK modulation the double carrier cyclic detector $\alpha= \pm 2 f_{\mathrm{c}}$ turns out to be the optimal detector [14]. However the phase-quadrature component present in QPSK modulation of the UMTS TDD signal, results in cancellation of the SCD at cyclic frequencies associated with the double carrier frequency. Thus, the detector of UMTS TDD cannot exploit this cyclostationary at $\alpha= \pm 2 f_{\mathrm{c}}$, and it is therefore necessary to resort to higher moments or to find other more suitable cyclic features in the base-band signal structure. The base-band cyclostationary properties come from the redundancy between frequency components separated by multiples of the symbol rate, i.e. $\alpha_{0}=1 /(S F \times T c)$, where $S F$ is the spreading factor and $T_{c}$ is the time chip. In UMTS standard $T_{c}=260 \mathrm{~ns}$.

\section{Exploring the Sensing Methods}

In order to evaluate the merits of the cyclostationary feature detector it is necessary to carry out an in-depth study of its performance. Figure 1 depicts the simulation chain used to test the UMTS signal detectors with AWGN, shadowing and multipath propagation conditions. The cyclostationary detector implementation uses a periodogram approach to estimate $S_{x}(f, \alpha)$ [15]. The FFT operations are computed over each set of $N$ samples, $N$ must be much higher than the period's cyclostationarity, so $N \gg S F$. After that, a time smoothing operation is done using an average operation over $K$ sets of $N$ samples. In this case, the observation time is Tobs $=N \times K \times \Lambda$, where $\Delta$ is the ADC sample period. However, in practical implementations the additional processing time required to compute $S_{x}(f, \alpha)$ must also be taken into account. Recall that $d$ is some decision statistic and $T H$ is a threshold level as discussed at the beginning of Section 2 .

In order to obtain the optimal detection statistic we should maximize the power of the spectral line $n$,

$$
Z_{o p t}(n)=\left\langle\int_{f} S_{x}\left(f, n \alpha_{0}\right) S_{y}^{*}\left(f, n \alpha_{0}\right) d f\right\rangle_{K}
$$

Where $<$. $>$ is an average in the time domain. However, the optimal detector can not be implemented without knowledge of the transmitted UMTS signal's phase, because $S_{y}$ depends on this phase. Our proposal is to use a sub optimal approach given by,

$$
Z(n)=\int_{f}\left|\left\langle S_{x}\left(f, n \alpha_{0}\right)\right\rangle_{K}\right|^{2}
$$

Instead of the power of the spectral line $n, Z(n)$, we chose as detection statistic the SNR measured in that spectral line. In the energy detector case the signal power is given by (8) with $n=0$ and the noise level is estimated measuring the signal power level in adjacent UMTS guard bands where only the noise is present. 


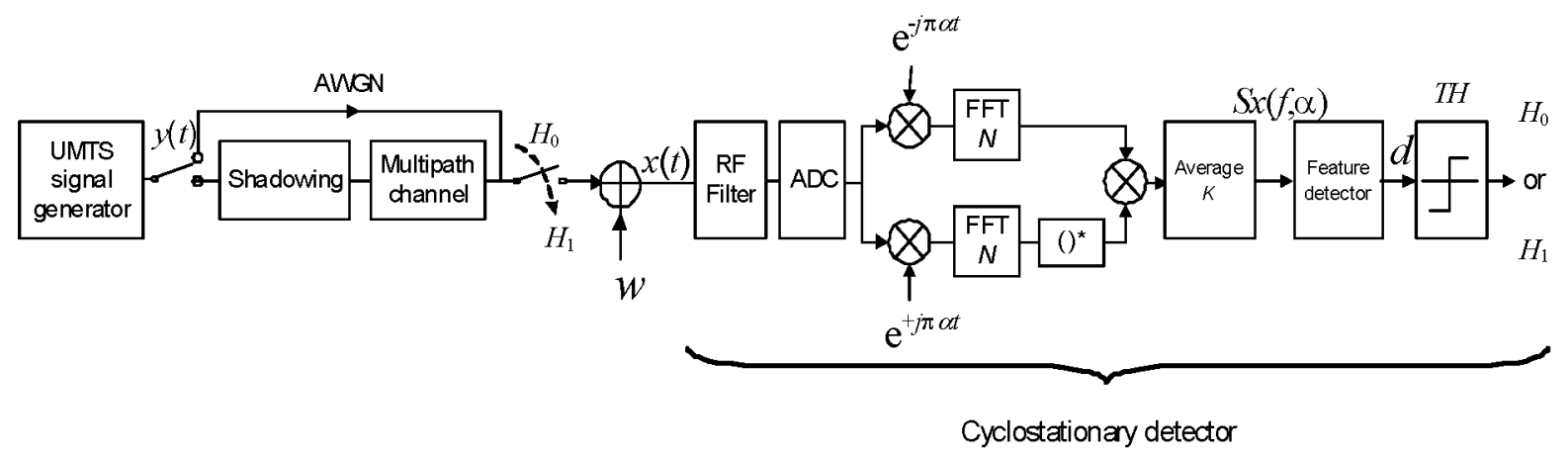

Fig. 1. Simulation Chain for testing of UMTS Signal Detectors.

On the other hand, in practical implementations of the cyclostationarity detector the Tobs is limited, so, the theoretical limit given by (6) cannot be reached and there will be always a noise floor of $S_{w}>0$, constant for all $\alpha \neq 0$. In order to estimate $S_{w}$, we can compute an average over $Q$ spectrum lines whose $\alpha \neq$ $n \alpha_{0}$, using, for instance, some intermediate frequencies between $n \alpha_{0}$ spectral lines. Therefore,

$$
\widetilde{S}_{w}=\frac{1}{Q} \sum_{q \neq n} \int_{f}\left|\left\langle S_{x}\left(f, \alpha_{q}\right)\right\rangle_{K}\right|^{2} d f
$$

and the detection statistic is given by,

$$
d(n)=\left(\frac{Z(n)}{\widetilde{S}_{w}}\right)^{1 / 2}<T
$$

It is still possible to increase the sensitivity of the cyclostationary detector with a new detection statistic $(D)$ which exploits others contributions coming from $M$ different cyclic frequencies,

$$
D=\sum_{n=1}^{M} d(n) \stackrel{<}{>} T H
$$

This detector is known as a multi-cycle detector and its complexity is $M$ times the complexity of the single cyclostationary detector, given by (10).

\section{Simulation Results}

In order to analyze the detection statistics of the UMTS signal for different radio channels and observation times, exhaustive simulations were carried out using the simulation chain presented in Figure 1 .

Table 1 summarizes the main simulation's parameters used. Without loss of generality, $N$ is considered equal to a length of an
UMTS time slot; however we do not assume any synchronisation between the OR and the UMTS terminal.

Table 1. Simulation's parameters.

\begin{tabular}{|l|l|}
\hline Asymmetry's service level & $\begin{array}{l}14 \text { time slot Downlink: } 1 \text { time } \\
\text { slot Uplink }\end{array}$ \\
\hline$S F$ & 16 \\
\hline Codes per time slot & 8 (half-load) \\
\hline$\alpha_{0}$ & $240 \mathrm{KHz}$ \\
\hline$\alpha_{q}$ & $Q=2 ; 120 \mathrm{KHz}$ and $360 \mathrm{KHz}$ \\
\hline$N$ & 2560 samples \\
\hline$\Delta$ & $T_{c}=260 \mathrm{~ns}$ \\
\hline$E[\mathrm{SNR}]$ & $-15 \mathrm{~dB}$ \\
\hline
\end{tabular}

In OR systems, both the OR and primary user can be in moving, which causes rapid variations of the radio channel conditions affecting the detection performance. Thus it is very important to study the effect of shadowing and multipath propagation in UMTS signals detection. Regarding the propagation channel we simulated the shadowing as log-normal distribution with a standard deviation, $\sigma_{d B}=4 \mathrm{~dB}[16]$. The multipath channel model is the UMTS Vehicular A with six Rayleigh components and mobile speed equal to $30 \mathrm{Km} / \mathrm{h}$ [17]. We consider Tobs of $30 \mathrm{~ms}$ and $300 \mathrm{~ms}$. The detection's performance in terms of ROC curve is presented in Figure 2. Plots for pure AWGN channel are also provided for comparison.

By comparing the AWGN curves with those corresponding to the multipath and shadowing cases it is clear, as expected, that sensing is harder in presence of multipath and shadowing. In order to balance the degradation in sensing ability it is possible to increase the observation time and/or increase the detector's complexity by using, for instance, a multi cyclic detector as defined in (11).

If we consider a critical specification for local sensing to be a $P d$ of $98 \%$, we can achieve this with $P f a=20 \%$ for Tobs $=300 \mathrm{~ms}$ or $P f a=35 \%$ for Tobs $=30 \mathrm{~ms}$. However, if we are able to double the detector's complexity $(M=2)$ it is possible to get achieve a $P d$ of $98 \%$ with only a $P f a$ of $10 \%$ and $T o b s=30 \mathrm{~ms}$. 


\section{Conclusions}

This paper focused on the use of cyclostationary feature detection for UMTS signals. Simulation results showed that using a local cyclostationary detector is possible to sense a week UMTS TDD signal, with $\mathrm{SNR}=-15 \mathrm{~dB}$, and keep a probability of detection equal to $98 \%$, even taking in account the shadowing and multipath propagation effects. With extra detection gain coming from possible collaborative sensing architectures and new cognitive functions we can envisage that UMTS TDD and OR can coexist without a degradation of the UMTS in real scenarios.

However in going forward with the work there are many more challenges. There are issues with the performance of the detector. In order to reach a probability of detection higher than $99.9 \%$ it will be necessary to extend this detector to collaborative decision features. One of the problems that can be studied is the optimal combination of sensing results coming from various OR terminals which have different sensitivities and observation times.

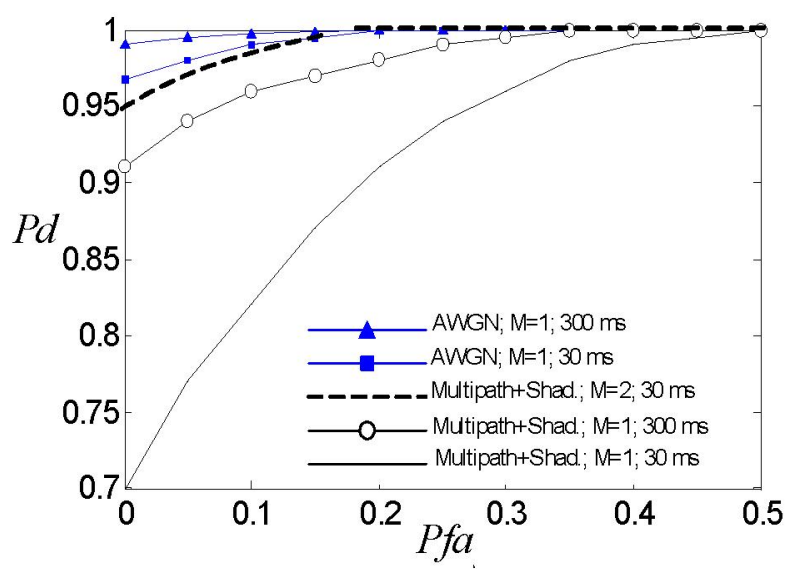

Fig. 2. ROC under log-normal shadowing and multipath propagation. AWGN is provided for comparison.

\section{References}

1. Diepenbeek C. Flexibility in frequency Management. 11th CEPT Conference, October 2004

2. Shared Spectrum Company. New York City Spectrum Occupancy Measurements September 2004, $\mathrm{http}: / / \mathrm{www}$. sharedspectrum.com/?section=presentations.

3. IEEE 802.22 Working Group http://www.ieee802.org/22.

4. Mitola J. Cognitive Radio for Flexible Multimedia Communications, MoMuC '99, pp. 3-10, 1999.

5. Sidenbladh T. Current status of $3 \mathrm{G}$ licensing. UMTS 2000 Conference, October 2000 and http://www.umts-forum.org.
6. Haardt M., Klein A., Koehn R., Oestreich S., Purat M., Sommer V. and Ulrich T. The TD-CDMA Based UTRA TDD mode. IEEE J. on Selected Areas in Comm., vol. 18, pp. 13751385, August 2000.

7. UMTS TDD Alliance. http://www.umtstdd.org.

8. Sonnenschein A. and Fishman P. Radiometric Detection of SpreadSpectrum Signals in Noise of Uncertain Power. IEEE Transactions on Aerospace Electronic Systems, vol. 28, no. 3, pp. 654-660, July 1992.

9. R. Tandra, A. Sahai. Fundamental limits on detection in low SNR under noise uncertainly. WirelessCom 2005, June 2005.

10. Gardner W., Spooner M. Signal Interception: Performance Advantages of Cyclic-Feature Detectors. IEEE Transactions on Communications, vol. 40, n0. 1, pp. 149-159, January 1992.

11. Ghasemi A., Sousa E. Collaborative Spectrum Sensing for Opportunistic Access in Fading Environments. DySPAN, November 2005.

12. Cabric D., Mishra S., Brodersen R. Implementation Issues in Spectrum Sensing for Cognitive Radio. Asilomar Conf. on Signals, Systems and Computers, Nov. 2004.

13. Weiss T., Jondral F. Spectrum Polling an innovative strategy for the enhancement of spectrum efficiency. IEEE Radio Communications, March 2004.

14. Gardner W., William A. Cyclostationarity in Communications and Signal Processing, IEEE PRESS, 1994.

15. Terrien C. Discrete Random Signals and Statistical Signal Processing, Prentice Hall, 1992.

16. Erceg V. An empirically based path loss model for wireless channels in suburban environments. IEEE Journal on Selected Areas in Communications, vol.17, pp. 1205-1211, July 1999.

17. ETSI. Universal Mobile Telecommunications System Selection procedures for the choice of radio transmission technologies of the UMTS. TR 101112, 1998, pp. 42-43. 